\title{
Correction to: Alcohol and Noncommunicable Disease Risk
}

\author{
Jürgen Rehm ${ }^{1,2,3,4,5,6} \cdot$ Omer S. M. Hasan ${ }^{1,5} \cdot$ Sameer Imtiaz ${ }^{1,3} \cdot$ Charlotte Probst $^{1,6} \cdot$ Michael Roerecke $^{1,5}$. \\ Kevin Shield ${ }^{1,5}$
}

Published online: 15 March 2018

(C) Springer International Publishing AG, part of Springer Nature 2018

\section{Correction to: Current Addiction Reports (2018) https://doi.org/10.1007/s40429-018-0189-8}

The original version of this article unfortunately contained a mistake. There are two instances where the content in Fig. 2 was changed to "[CATEGORY NAME][PERCENTAGE]," instead of what actually should be shown. With this, the original article was corrected and the correct Fig. 2 is now presented in here.

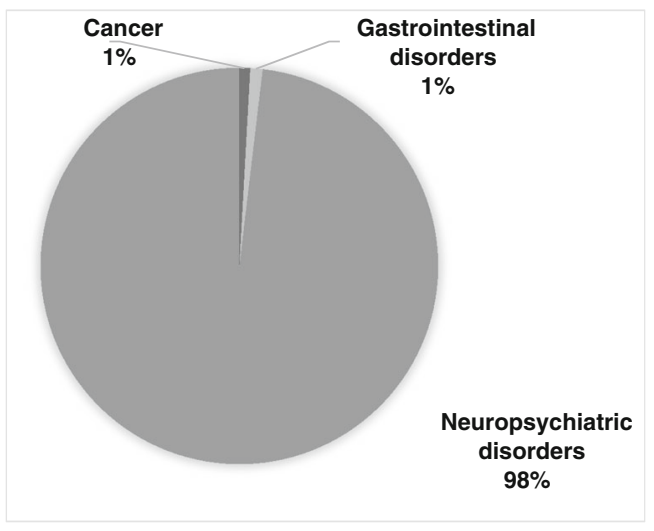

The online version of the original article can be found at https://doi.org/ 10.1007/s40429-018-0189-8

Jürgen Rehm

jtrehm@gmail.com

1 Institute for Mental Health Policy Research, Centre for Addiction and Mental Health (CAMH), 33 Russell Street, Toronto, ON M5S 2S1, Canada

2 Campbell Family Mental Health Research Institute, CAMH, 250 College Street, Toronto, ON M5T 1R8, Canada

3 Institute of Medical Science (IMS), University of Toronto, Medical Sciences Building, 1 King's College Circle, Room 2374, Toronto, ON M5S 1A8, Canada

4 Department of Psychiatry, University of Toronto, 250 College Street, 8th Floor, Toronto, ON M5T 1R8, Canada

5 Dalla Lana School of Public Health, University of Toronto, 155 College Street, 6th Floor, Toronto, ON M5T 3M7, Canada

6 Institute for Clinical Psychology and Psychotherapy, TU Dresden, Chemnitzer Str. 46, 01187 Dresden, Germany 